\title{
Incidental Computer Tomography Radiologic Findings through Research Participation in the North Texas Healthy Heart Study
}

\author{
Anna Espinoza, MD, Kendra Malone, BS, Elizabeth Balyakina, MS, MPH, \\ Kimberly G. Fulda, DrPH, and Roberto Cardarelli, DO, MPH
}

Background: Although variation exists in the classification and practice of managing clinical findings in research, emerging views suggest that researchers bear some responsibility in the management of incidental findings. This study contributes to the documentation of the population characteristics and prevalence of medical findings incidental to research participation, specifically findings related to coronary calcium scores and computed tomography (CT) scans that investigated cardiovascular disparities in an asymptomatic population.

Methods: A total of 571 asymptomatic adult participants were recruited in the North Texas Healthy Heart Study. Participants completed a 16-slice CT scan of the heart and abdomen. Findings of radiology reports and 3 years of follow-up documentation were reviewed.

Results: A total of 246 clinically apparent findings were identified in 169 asymptomatic participants (32.9\% of participants who completed a CT scan). Another 245 participants (48\%) had findings of unknown significance, a total of 307 findings. At least 4 cases in this study led to a clinically significant intervention.

Conclusion: Although CT scans were completed for research purposes, study procedures resulted in the diagnosis and treatment of individuals who were previously asymptomatic. Potential clinical benefits in imaging research are moderated by considerations regarding possible harm and costs resulting from uncertain findings and the use of CT scans for nonclinical purposes. The continued development of protocols for the handling of incidental findings in research and the establishment of guidelines are needed to ensure that research procedures mirror the best interests of participants. ( $\mathrm{J}$ Am Board Fam Med 2014;27:314-320.)

Keywords: Computed Tomography, Incidental Findings, Medical Ethics, Practice-based Research Networks, Research Subjects

Recent advances in computed tomography (CT) have allowed the production of more defined images in shorter times. The availability of more advanced CT has resulted in increased medical use, with consequent increases in radiation exposure

This article was externally peer reviewed.

Submitted 6 September 2013; revised 8 January 2014; accepted 13 January 2014.

From the Primary Care Research Center/Texas Prevention Institute (AE, KM, EB, KGF, RC), the Department of Family Medicine (KGF), and the Department of Family and Community Medicine (RC), University of Kentucky College of Medicine, Lexington.

Funding: This study was supported by grant no. P20MD001633 from the National Center on Minority Health and Health Disparities and the National Institutes of Health Loan Repayment Program. among patients. ${ }^{1-3}$ The use of CT in clinical settings has been examined in the literature, describing both the clinical benefit of using less invasive techniques and identifying increased radiation-associated cancer risk with imaging of different parts of the body. ${ }^{4-7}$ The use of CT for research purposes and the resulting incidental findings (IFs)

Conflict of interest: none declared.

Disclaimer: This content is solely the responsibility of the authors and does not necessarily represent the official views of the National Institute on Minority Health and Health Disparities or the National Institutes of Health. Corresponding author: Roberto Cardarelli, DO, MPH, Department of Family and Community Medicine, University of Kentucky, 740 S. Limestone K311, Lexington, KY 40536 (E-mail: roberto.cardarelli@uky.edu). 
have been less closely considered, with recent calls for the documentation of the prevalence of IFs in different research settings and guidance on how to manage findings. ${ }^{8-12}$

Medical practice is premised on the provision of interventions designed to improve the well-being of a patient with a reasonable expectation of success. ${ }^{13,14}$ In contrast, research is characterized by activities intended to contribute to new and generalizable knowledge, presenting a different basis from which to consider ethics regarding medical testing provided during the course of study procedures. Some studies show that detection of IFs may lead to earlier diagnoses, whereas others document increased costs related to the generation of a large number of findings with unknown significance and the possibility of further unnecessary testing. ${ }^{9,15-18}$ Follow-up to IFs in research settings is complicated by the lack of institutional and federal regulations on how to manage such findings in research and unbudgeted costs associated with management. ${ }^{8,9,18,19}$ In addition, IFs may be difficult to follow because findings are typically managed independent of or tangential to research protocols. ${ }^{9}$

Although variation in the classification and practice of managing clinical findings in research exists, emerging views suggest that researchers bear some responsibility in the management of IFs. ${ }^{9,20,21}$ This study contributes to the documentation of the population characteristics and prevalence of medical findings incidental to research participation, specifically findings related to coronary calcium scores and CT findings resulting from participation in a primary care practice-based research network study that investigated disparities in cardiovascular health outcomes. Although coronary artery calcium is an established marker of increased cardiovascular risk, the indications for which testing is recommended continues to be a subject of debate, in particular with regard to asymptomatic patients. ${ }^{22}$ Case vignettes are provided here to qualitatively illustrate follow-up and clinical management procedures used when clinically apparent findings were identified during the course of the study.

\section{Methods}

\section{Study Procedures}

The North Texas Healthy Heart Study recruited a purposive sample of 571 subjects and assessed the association of psychosocial and metabolic factors among 3 racial/ethnic groups to examine factors related to disparities in cardiovascular health. Participants were recruited from the North Texas (NorTex) primary care practice-based research network from April 2006 to June 2008, with a repeat visit after 2 years and yearly follow-up calls for 3 years. Participants consisted of men and women 45 years of age and older and self-reported as non-Hispanic white, non-Hispanic African American, or Hispanic. Exclusion criteria included a self-reported history of cardiovascular disease, renal failure, and/or liver failure. Study procedures were completed at the University of North Texas Health Science Center at Fort Worth, and were approved by the institutional review boards at University of North Texas Health Science Center and John Peter Smith Hospital.

Multislice CT scans (MSCTs) of the heart and abdomen were performed for each participant using a 16-slice CT scanner (Aquilion 16, model TSX-101A; Toshiba America Medical Systems, Inc., Tustin, CA) to obtain a coronary calcium and visceral adipose tissue score. Coronary artery calcium (CAC) scores were measured by taking images every $3 \mathrm{~mm}$ from the carina to the base of the heart. The scanner was open, and no oral or intravenous contrast media were used. A radiologist, who was blinded to the study results, interpreted all scans. Eight axial CT images of the abdomen were obtained from each subject for abdominal visceral adipose tissue measurements; slice 6 was centered on the L4-L5 interspace.

\section{Demographic and Health-Related Measures}

Demographic and selected health behavior information were collected using standardized questions from the Behavioral Risk factor Surveillance System. Age was registered as a continuous variable. Selfreported race/ethnicity was categorized as nonHispanic white, non-Hispanic African American, and Hispanic. Education was coded as less than high school, high school graduate, and some college or higher. Smoking status was assessed using the question, "Have you smoked at least 100 cigarettes in your entire life?" and was coded as a dichotomous variable. Family history of heart disease was similarly assessed using the question, "Did any of your first-degree family members ever have a heart attack or blocked arteries?"

Hypertension was considered present if the participant had an average blood pressure $\geq 140 / 90$ 
$\mathrm{mmHg}$, reported a previous diagnosis of hypertension, or was taking antihypertensive medication. Diabetes was considered present if the participant had a fasting blood glucose concentration $\geq 126$ $\mathrm{mg} / \mathrm{dL}$, reported a previous diagnosis of diabetes, or was taking diabetic medication. Hyperlipidemia was considered present if the participant showed low-density lipoprotein levels $\geq 160 \mathrm{mg} / \mathrm{dL}$, reported a previous diagnosis of hyperlipidemia, or was taking a lipid-lowering medication.

\section{Analysis of Incidental Findings}

Radiology reports and 3 years of follow-up documentation were reviewed for findings and coded according to clinically apparent findings and findings of unknown significance. Clinically apparent findings were defined as CT results that yielded diagnoses with a known definite course of treatment or management. Findings of unknown significance were defined as CT findings whose prognoses were indeterminate from radiology findings, study follow-up, or other study documentation and would normally require further diagnostics or assessments to yield a final diagnosis.

Follow-up documentation was assessed for resolution of responses to the following questions: "Were you hospitalized, and, if yes, for what?" "Were you diagnosed with any serious medical conditions, and, if yes, for what?" and "Did you have more health problems this past year?" Surveys also were reviewed for documentation of a heart attack, stroke, heart blockages, ministroke, or diagnosis of heart failure during the 3-year follow-up period. Study progress notes, documented patient communication such as letters and phone conversations, insurance status, and presence of a primary care physician also were reviewed to determine how findings and their resolutions were managed.

\section{Results}

Among 571 study participants, 513 completed a CT scan. Of participants who completed a scan, $62 \%$ were female (Table 1). Participants were, on average, 55.1 years old, and $57.7 \%$ had completed some college or more. Participants were non-Hispanic white (28.1\%), African American (34.7\%), and Hispanic $(37.2 \%)$, and $42 \%$ had smoked at least 100 cigarettes in their life. Of participants, $51 \%$ had hypertension, $51.7 \%$ had hyperlipidemia, and $18.9 \%$ had diabetes; $34.9 \%$ showed some cor-
Table 1. Characteristics of North Texas Healthy Heart Study Participants, Fort Worth, Texas, 2006 to 2008 $(\mathrm{n}=\mathbf{5 1 3})$

\begin{tabular}{|c|c|}
\hline Variable & Participants* \\
\hline Age (years), mean (SD) & $55.1(8.0)$ \\
\hline \multicolumn{2}{|l|}{ Sex } \\
\hline Female & $320(62.4)$ \\
\hline Men & $193(37.6)$ \\
\hline \multicolumn{2}{|l|}{ Race/ethnicity } \\
\hline Non-Hispanic white & $144(28.1)$ \\
\hline Non-Hispanic black & $178(34.7)$ \\
\hline Hispanic & $191(37.2)$ \\
\hline \multicolumn{2}{|l|}{ Education } \\
\hline Less than high school & $113(22.0)$ \\
\hline High school graduate/GED & $104(20.3)$ \\
\hline Some college or higher & $296(57.7)$ \\
\hline \multicolumn{2}{|c|}{ Smoked $\geq 100$ cigarettes in one's life } \\
\hline Yes & $217(42.3)$ \\
\hline No & $289(56.3)$ \\
\hline \multicolumn{2}{|l|}{ Diabetes mellitus status } \\
\hline Yes & $97(18.9)$ \\
\hline No & $393(76.6)$ \\
\hline \multicolumn{2}{|l|}{ Hypertension status } \\
\hline Yes & $262(51.1)$ \\
\hline No & $127(24.8)$ \\
\hline \multicolumn{2}{|l|}{ Hyperlipidemia status } \\
\hline Yes & $265(51.7)$ \\
\hline No & $232(45.2)$ \\
\hline \multicolumn{2}{|c|}{$\begin{array}{l}\text { First-degree relative with history of coronary } \\
\text { heart disease }\end{array}$} \\
\hline Yes & $251(48.9)$ \\
\hline No & $248(48.3)$ \\
\hline
\end{tabular}

Data are n (\%) unless otherwise indicated. *May not add up to 513 participants because of missing data.

$\mathrm{SD}$, standard deviation.

onary calcification (CAC >0), and $48.9 \%$ had a family history of heart disease. Characteristics of those who did not complete a CT scan were similar to those who completed a scan.

A total of 246 clinically apparent findings were identified in 169 asymptomatic participants (32.9\% of participants who completed a CT scan). The 3 most common findings were osteoarthritis, hernia, and fatty infiltration of the liver (Table 2). Hernia includes hiatal, periumbilical, paraumbilical, and abdominal hernias. Other common findings included diverticulosis, ecstasia of the thoracic aorta or aortic root, and nephrolithiasis. Almost 307 findings of unknown significance, such as pulmonary nodules, liver lesions, and questionable lymph nodes, were identified in 245 participants (48\%) 
Table 2. Clinically Apparent Computed Tomography Findings in the North Texas Healthy Heart Study $(n=246)$

\begin{tabular}{lr}
\hline Findings & No. \\
\hline Osteoarthritis & 134 \\
Hernia & 30 \\
Fatty infiltration of the liver & 27 \\
Diverticulosis & 16 \\
Ecstasia & 12 \\
Cardiomegaly & 10 \\
Nephrolithiasis & 9 \\
Cholelithiasis & 7 \\
Leukemia & 1 \\
\hline
\end{tabular}

(Table 3). A total of 22 participants had a CAC score $>500$, and 164 subjects had a CAC between 0 and 500 (Figure 1), whereas 324 participants had a CAC score of 0 arterial calcification.

At least 4 cases in this study required a clinically significant intervention. The following 4 case vignettes were selected as examples of the steps taken during the course of study procedures to ensure appropriate treatment and referral of participants when clinical findings were discovered.

\section{Case 1}

A 61-year-old Hispanic man had a calcium score of 2384, indicating extensive atherosclerotic plaque

Table 3. Computed Tomography Findings of Unknown Significance in the North Texas Healthy Heart Study

$(\mathrm{n}=307)$

\begin{tabular}{lr}
\hline Findings & No. \\
\hline Pulmonary nodules & 151 \\
Liver lesions (possible cysts or hemangiomas) & 33 \\
Questionable lymph nodes & 5 \\
Emphysematous change & 11 \\
Gynecological findings* & 17 \\
Gallbladder findings $^{\dagger}$ & 9 \\
Bronchiectasis $_{\text {Renal cysts }}$ & 8 \\
Pulmonary cysts/indeterminate lesion & 11 \\
Breast lesions & 5 \\
Other & 5 \\
\hline
\end{tabular}

*Includes enlarged uterus or ovaries, uterine mass, uterine or ovarian cysts, and ovarian vein phlebolith; excludes atelectasis $(\mathrm{n}=132)$.

${ }^{\dagger}$ Includes irregularity of gallbladder, contracted gallbladder, dilated gallbladder, probable gallstones, and possible gallbladder sludge.
Figure 1. Coronary calcium scores of participants $(n=$ 513) in the North Texas Healthy Heart Study. *May not add up to 513 participants because of missing values.

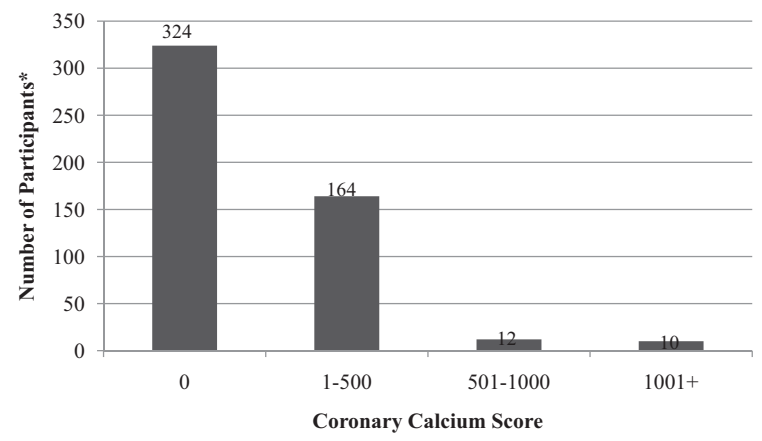

and a high likelihood of at least one significant coronary artery stenosis. The participant was referred to his primary care provider and was subsequently referred to a cardiologist. He underwent an elective procedure to place stents in 4 coronary arteries. The procedure was well tolerated and had no complications, and the patient was released from the hospital within 36 hours. The participant was asymptomatic before this study's procedures and completed them in May 2007; he underwent coronary stent placement in August 2007.

\section{Case 2}

A 66-year-old married, non-Hispanic white man had a calcium score of 0 . The participant's CT scan showed a diffuse enlarged spleen measuring 15.9 $\mathrm{cm}$ (anterior to posterior). He was referred to his primary care physician and was later diagnosed with leukemia. At the first year follow-up, the participant had relocated outside of Texas and per patient report was free of cancer.

\section{Case 3}

A 46-year-old non-Hispanic white woman had a calcium score of 0 but a significant history of severe menorrhagia and anemia. CT of the abdomen showed an enlarged uterus or abnormal uterine mass requiring further evaluation with a pelvic sonogram. The participant was referred to her primary care physician, which eventually resulted in a total hysterectomy 3 months later (as documented during study follow-up). The patient self-reported that the pathology was a uterine fibroid.

\section{Case 4}

A 67-year-old Hispanic man had a calcium score of 3987 , indicating the presence of extensive athero- 
sclerotic plaque (at least one significant coronary stenosis). Mild calcifications of the thoracic aorta also were identified. The study's principal investigator (RC) notified the participant and the subject's primary care physician. During the first year follow-up phone call, the subject self-reported that he underwent a coronary artery bypass graft surgery several months after the finding.

\section{Discussion}

Orme and colleagues ${ }^{10}$ indicate that the evaluation of imaging completed for research purposes leads to a high number of IFs that result in important clinical benefits to a small but significant minority of participants. Of the 513 participants who completed CT scans in the present study, there were at least 4 known cases that resulted in significant clinical intervention. In comparison, 1090 routine CT scans with no contrast are estimated to result in one radiation-induced cancer among women 60 years of age; 2080 routine CT scans are estimated to lead to the development of one cancer among men aged 60 years. $^{2}$ In this study, the prevalence of clinically apparent findings leading to a clinical intervention suggests that the benefits may outweigh the radiation risks typically associated with imaging. ${ }^{1-3}$ Moreover, the chest MSCT used in the study exposes an individual to approximately $3 \mathrm{mSv}$ of radiation, compared with $7 \mathrm{mSv}$ from a traditional chest CT scan, further conferring less risk. ${ }^{23} \mathrm{Al}-$ though CT scans were completed for research purposes, study procedures resulted in the diagnosis and treatment of individuals who were previously asymptomatic.

Potential clinical benefits in imaging research are moderated by considerations regarding possible harms and costs resulting from uncertain findings and the use of CT scans for nonclinical purposes. ${ }^{9,15-17}$ Clinically, the use of CT scans to test for coronary calcium is not recommended for asymptomatic patients and can result in anxiety and further unnecessary testing if findings are in fact benign. ${ }^{24}$ The consent process in research settings, therefore, plays an important role in conveying both the risks and benefits of clinical tests such as CT that are provided during the course of study procedures. Aldington and colleagues ${ }^{9}$ recommend that the potential for IFs should be disclosed during informed consent and how possible findings will be managed should be identified. The Presidential
Commission for the Study of Bioethical Issues recommends that clinicians engage in shared decision making with participants about the scope of findings that will be probed and subsequent steps that will be taken. Shared decision making should reflect the best interests of the participant. ${ }^{21}$ Consent must also address the difference between participating in research and seeking medical testing for clinical care purposes, given the differences in the underlying ethical considerations in providing testing in clinical and research settings ${ }^{13,14}$ and, as this study demonstrates, the potential for clinically significant findings.

In a study of CT chest scans conducted while examining the pulmonary effects of smoking, 19.9\% of participants required referral or follow-up as a result of incidental study findings. ${ }^{9}$ Varying incidence of IFs has been observed in studies of CT screenings for heart disease, lung cancer, and diagnosis of renal stones. ${ }^{25-27}$ In a review of chest CT screening studies, $7.7 \%$ of patients undergoing screening for coronary artery disease and $14.2 \%$ of patients undergoing screening for lung cancer had IFs requiring additional follow-up. ${ }^{25}$ In one study, $45 \%$ of noncontrast, helical, abdominal CT scans conducted in an emergency department setting for the diagnosis of renal stones resulted in IFs, of which $23.1 \%$ were considered moderate or severe. ${ }^{26}$ In the current study, approximately $60 \%$ of participants had findings of unknown significance and $32.9 \%$ of participants had clinically apparent findings, with combined results for both chest and abdominal MSCT. In a study of patients who underwent elective multidetector CT, $43 \%$ had IFs, compared with $33 \%$ in the current study. ${ }^{27}$ The current study, however, included only asymptomatic individuals with no known cardiac disease. Comparison between studies is complicated by differences in the categorization of IFs, the body region for which scanning procedures were completed, and follow-up procedures. In addition, the number of IFs identified in the current study may have been higher if contrast or a greater number of slices had been used.

Coordination of the study team with primary care physicians and a federally qualified medical clinic were necessary to ensure appropriate referral and follow-up of study participants. Findings of high potential clinical significance were verbally communicated to participants by the physician principal investigator (RC). Referral to a primary 
care physician was the primary method used to manage clinical IFs. When a participant was not insured, they were provided with information about a federally qualified medical clinic. Follow-up and documentation of participant outcomes was possible because of the longitudinal nature of the research. However, the study's longitudinal surveys included general questions about hospitalizations and clinical diagnoses since the previous study visit. The questions were not specific to the CT findings. Including follow-up questions specific to incidental findings may provide more detailed information.

Clinical responsibility may conflict with participants' informed preferences; such is the case when participants decline to learn information related to the primary purpose of the study procedures. In such a case, the participant should forego the procedure. ${ }^{21}$ Clinical responsibility in research may be constrained by difficulties in participant follow-up, such as in cases where participants relocate or change contact information, as was illustrated in case 2. Follow-up may be further complicated in studies that do not follow a longitudinal design. Other barriers to follow-up identified in the literature include a lack of funding for the management of findings in study protocols and the expectation placed on potentially nonclinician researchers to process clinical findings. ${ }^{9,12}$

Findings of this study are specific to an older asymptomatic population. Research procedures that include medical testing should emphasize the difference between participating in research and seeking medical care, especially in cases where research and practice may occur in the same setting. While this study focuses on the use of CT, the considerations raised in this study may apply to other forms of clinical testing and care provided during research procedures and provide a basis for further consideration of the intersection between research and clinical practice. Current practices for managing IFs in research may not reflect the best interests of study volunteers. ${ }^{28,29}$ The continued development of protocols for handling IFs in research and the establishment of guidelines are needed to ensure that research procedures mirror the best interests of participants. ${ }^{8,12,30}$

The authors are grateful for the assistance of Joan Carroll, $\mathrm{PhD}$, Ana L. Chiapa, Mayra Rodriguez, Lorna Brooks, and the Primary Care Research Center staff of the University of North
Texas Health Science Center. We also thank the clinicians of the North Texas Primary Care Research Network, who helped recruit study participants.

\section{References}

1. Brenner DJ, Elliston CD. Estimated radiation risks potentially associated with full-body CT screening. Radiology 2004;232:735-8.

2. Smith-Bindman R, Lipson J, Marcus R, et al. Radiation dose associated with common computed tomography examinations and the associated lifetime attributable risk of cancer. Arch Intern Med 2009; 169:2078-86.

3. Haaga JR. Radiation dose management: weighing risk versus benefit. AJR Am J Roentgenol 2001;177: 289-91.

4. Lee CI, Haims AH, Monico EP, Brink JA, Forman HP. Diagnostic CT scans: assessment of patient, physician, and radiologist awareness of radiation dose and possible risks. Radiology 2004;231:393-8.

5. Brenner DJ. Slowing the increase in the population dose resulting from CT scans. Radiat Res 2010;174: 809-15.

6. Dougeni E, Faulkner K, Panayiotakis G. A review of patient dose and optimisation methods in adult and paediatric CT scanning. Eur J Radiol 2012;81: e665-83.

7. Bach PB, Jett JR, Pastorino U, Tockman MS, Swensen SJ, Begg CB. Computed tomography screening and lung cancer outcomes. JAMA 2007; 297:953-61.

8. Wolf SM, Paradise J, Caga-anan C. The law of incidental findings in human subjects research: establishing researchers' duties. J Law Med Ethics 2008;36:361-83.

9. Aldington S, Shirtcliffe P, Nowitz M, et al. Incidental findings from lung CT scans: implications for research. J Med Imaging Radiat Oncol 2011;55: $20-5$.

10. Orme NM, Fletcher JG, Siddiki HA, et al. Incidental findings in imaging research: evaluating incidence, benefit, and burden. Arch Intern Med 2010;170: 1525-32.

11. Seki A, Uchiyama H, Fukushi T, Sakura O, Tatsuya $\mathrm{K}$. Incidental findings of brain magnetic resonance imaging study in a pediatric cohort in Japan and recommendation for a model management protocol. J Epidemiol 2010;20(Suppl 2):S498-504.

12. Wolf SM, Lawrenz FP, Nelson CA, et al. Managing incidental findings in human subjects research: analysis and recommendations. J Law Med Ethics 2008; 36:219-48.

13. Jefford M, Moore R. Improvement of informed consent and the quality of consent documents. Lancet Oncol 2008;9:485-93.

14. The Belmont report: ethical principles and guidelines for the protection of human subjects of research. Washington, DC: Department of Health, 
Education, and Welfare, U.S. Department of Health and Human Services; 1979.

15. Hara AK. Extracolonic findings at CT colonography. Semin Ultrasound CT MR 2005;26:24-7.

16. Siddiki H, Fletcher JG, McFarland B, et al. Incidental findings in CT colonography: literature review and survey of current research practice. J Law Med Ethics 2008;36:320-31.

17. Stone JH. Incidentalomas-clinical correlation and translational science required. N Engl J Med 2006; 354:2748-9.

18. Presidential Commission for the Study of Bioethical Issues. Bioethics Commission on Incidental Findings: anticipate and communicate. Presss Release. December 12, 2013. Available from: http://bioethics.gov/node/ 3186. Accessed March 7, 2014.

19. Wolf SM. The role of law in the debate over return of research results and incidental findings: the challenge of developing law for translational science. Minn J Law Sci Technol 2012;13:435-48.

20. Richardson HS. Incidental findings and ancillarycare obligations. J Law Med Ethics 2008;36:256-70.

21. Gutmann A. Policy forum ethics: the Bioethics Commission on Incidental Findings. Science 2013; 342:1321-3.

22. Goel R, Garg P, Achenbach S, et al. Coronary artery calcification and coronary atherosclerotic disease. Cardiol Clin 2012;30:19-47.

23. Safety: radiation exposure in x-ray examinations. 2009. Radiological Society of North America. Available from: http://oralcancerfoundation.org/facts/pdf/x-ray_safety. pdf. Accessed March 20, 2014.
24. National Cholesterol Education Program (NCEP) Expert Panel on Detection, Evaluation, and Treatment of High Blood Cholesterol in Adults (Adult Treatment Panel III). Third report of the $\mathrm{Na}$ tional Cholesterol Education Program (NCEP) Expert Panel on Detection, Evaluation, and Treatment of High Blood Cholesterol in Adults (Adult Treatment Panel III): final report. Circulation 2002;106:3143-421.

25. Jacobs PC, Mali WP, Grobbee DE, van der Graaf Y. Prevalence of incidental findings in computed tomographic screening of the chest: a systematic review. J Comput Assist Tomogr 2008;32:214-21.

26. Messersmith WA, Brown DFM, Barry MJ. The prevalence and implications of incidental findings on ED abdominal CT scans. Am J Emerg Med 2001; 19:479-81.

27. Lee CI, Tsai EB, Sigal BM, Plevritis SK, Garber AM, Rubin GD. Incidental extracardiac findings at coronary CT: clinical and economic impact. AJR Am J Roentgenol 2010;194:1531-8.

28. Booth TC, Jackson A, Wardlaw JM, Taylor SA, Waldman $\mathrm{AD}$. Incidental findings found in "healthy" volunteers during imaging performed for research: current legal and ethical implications. Br J Radiol 2010;83: 456-65.

29. Booth TC, Waldman AD, Wardlaw JM, Taylor SA, Jackson A. Management of incidental findings during imaging research in "healthy" volunteers: current UK practice. Br J Radiol 2012;85:11-21.

30. Cramer SC, Wu J, Hanson JA, et al. A system for addressing incidental findings in neuroimaging research. NeuroImage 2011;55:1020-3. 